The International Journal of Indian Psychology

ISSN 2348-5396 (e) | ISSN: 2349-3429 (p)

Volume 4, Issue 1, No. 80, DIP: 18.01.106/20160401

ISBN: 978-1-365-57867-0

http://www.ijip.in | October-December, 2016

\title{
Anxiety among Adolscents and Adults
}

\author{
Pavithraraj $^{1 *}$, Dr V Chandramohan ${ }^{2}$
}

\section{ABSTRACT}

The purpose of the study was to measure the anxiety level among adolescent group and adult group. It was hypothesized that adolescent group anxiety level is higher in adolescent group as compared to adult group. The sample used in the study was 30 male subjects out of which 15 were adolescents and 15 adults of age group between 18 to 25. The study was conducted on medical students of IIAMR.

Between group design was used for the study. The tools used for the study was Self Analysis Form [IPAT] developed by Kurg, Scheier and R.B.Cattell the questionnaire had 40 questions with 3 alternatives $\mathrm{A}, \mathrm{B}, \mathrm{C}$. The data was analyzed using ' $\mathrm{t}$ ' test the obtained ' $\mathrm{t}$ ' scores were 1.29 which is not significant at both 0.05 and 0.01 level.

Thus the adolescent group proves the hypothesis which states that "anxiety level is higher in adolescent group as compared to adult group”.

\section{Keywords: Anxiety, Science Students, Adolescents \& Adults}

The importance of psychological adjustment of children with chronic diseases has been emphasized (Stam, Grootenhuis, Caron, \& Last, 2006). In particular, anxiety has been increasingly discussed and recognized by a number of researchers as one of the most significant indicators of psychological adjustment to chronic disease in children (Lähteenmäki, Sjöblom, Korhonen, \&Salmi, 2004; Moore \& Mosher, 1997). For example, anxiety was used as an indicator of coping in adolescents with chronic pain (Claar, Baber, Simons, Logan, \& Walker, 2008), children and adolescents with sickle cell disease (Benton, Ifeagwu, \& Smith-Whitley, 2007), inflammatory bowel disease (Mackner, Crandall, \& Szigethy, 2006), juvenile arthritis (Mullick, Nahar, \& Haq, 2005), cancer, (Schultz, et al., 2007; Stam, Grootenhuis, \& Last, 2001), and children with asthma (Akçakaya, Aydogan, Hassanzadeh, Camcioglu, \& Cokugras, 2003).

\footnotetext{
${ }^{1}$ Research Scholar, Department of Psychology, Bharathiar University, Coimbatore \& Assistant Professor of Psychology, Mount Carmel college, Bangalore, India

${ }^{2}$ Research Supervisor, Reader in Psychology, Institute of Aerospace Medicine, IAF, Vimanapura (PO), Bangalore, India

*Responding Author

(C) 2016 Pavithraraj, Chandramohan V; licensee IJIP. This is an Open Access Research distributed under the terms of the Creative Commons Attribution License (http://creativecommons.org/licenses/by/2.0), which permits unrestricted use, distribution, and reproduction in any Medium, provided the original work is properly cited.
} 


\section{Anxiety among Adolscents and Adults}

While numerous forms of anxiety measurements are available, discussions regarding strengths and weaknesses of each measurement approach have been scarce. The purpose of this paper is to discuss a variety of approaches used by researchers to measure anxiety in children. Such information would help researchers to adequately identify and select relevant anxiety measures for the purpose of their future studies.

\section{CONCEPTUAL BASIS OF ANXIETY MEASURES}

Since Freud's conceptualization of anxiety-neurosis in 1894 as a discrete clinical syndrome, anxiety has been measured within the context of psychological Received: February 23, 2009 Revised: March 17, 2009 Accepted: April 27, 2009 theory (Spielberger, 1966). Even though there is a lack of agreement regarding the nature of anxiety, most researchers have developed their measures based on Freudian theory, though recently there has been an increasing discussion of physiological theories.

Psychological and physiological theories of anxiety Freud first attempted to explicate the concept of anxiety in the context of science (Spielberger, 1966).

According to Freud (1936), anxiety is regarded as an unpleasant affective state or condition, which is characterized by all that is covered by the word, 'nervousness'. Freud conceived of anxiety as a signal indicating the presence of a dangerous situation and differentiated between objective anxiety and neurotic anxiety largely on the basis of whether the source of the danger was from the external world or from internal impulses. Based on Freudian theory, objective anxiety involves a complex internal reaction to anticipated injury or harm from some external danger. With objective anxiety, the intensity of the anxiety reaction is proportional to the magnitude of the external danger that evokes it, the greater the external danger, the stronger the perceived threat, the more intense the resulting reaction. Neurotic anxiety is characterized by feelings of apprehension and physiological arousal (Freud). However, the source of danger is internal, and this source is not consciously perceived because it has been repressed. As an extension of the Freudian theory, May (1950) described anxiety as the apprehension cued off by a threat to some value which the individual holds essential to one's existence as a personality. According to May, the particular events or stimuli which evoked anxiety are largely determined by learning rather than impulses. An anxiety reaction is normal if it is proportionate to the objective danger and does not involve repression or other defense mechanism. Neurotic anxiety reactions are disproportionate to the objective danger and involve repression and neurotic defenses. Anxiety is also viewed as an intensely unpleasant state of tension arising from experiencing disapproval in interpersonal relations. For example, through an empathic linkage between an infant and its mother, the tension of anxiety, when present in the mothering one, induces anxiety in the infant (Sullivan, 1953). Once aroused, anxiety distorts the individual's perception of reality and limits the ranges of stimuli that are perceived. Today, anxiety is viewed as an unpleasant emotional state, which is characterized by subjective feelings of tension,

(C) The International Journal of Indian Psychology, ISSN 2348-5396 (e)| ISSN: 2349-3429 (p) | 51 


\section{Anxiety among Adolscents and Adults}

apprehension, and worry, and by activation or arousal of the autonomic nervous system (Bourne, 2006; Watson \& Kendall, 1989;Wesley, 1988), mostly consistent with Freud's conceptualization. Recently, there has been increasing discussion of physiological theories behind anxiety. These theories explain that individuals with anxiety disorder experience heightened physiological arousal when they encounter stressors or social situations, which they interpret as an indication of danger or anxiety (Gerlach, Mourlane, \&Rist, 2004). Anderson and Hope (2009) also noted that such an interpretation of physiological arousal leads to increased symptoms of anxiety (e.g. racing heart or blushing) among individuals with anxiety disorder. Furthermore, perceptions about the dangerousness of such physiological arousal may maintain anxiety symptoms as individuals learn to avoid threatening or stressful situations in order to evade such physiological arousal (Anderson \& Hope).

\section{REVIEW OF LITERATURE}

Hsiao C-C. (2012) the study examining the relationships among coping, anxiety and resilience and to identify predictors of anxiety and resilience in adolescents undergoing cancer treatment. Cross-sectional study. Adolescents $(n=131)$ recruited from three medical centers between 2010-2011. The eligible participants were diagnosed with cancer, without mental disease and receiving chemotherapy. Participants were assessed with the pediatric cancer coping scale, revised children's manifest anxiety scale, second edition, and the Haase adolescent resilience in illness scale .Cognitive coping and defensive coping are predictors for the level of anxiety and resilience in adolescents undergoing cancer treatment. Health providers should evaluate coping behavior in patients and work towards a cognitive and problem-oriented coping style that will benefit the patient's mental health during treatment.

Jaime lin (2012). This study was conducted between August 2009 and August 2010; all patients were recruited in schools, pediatric or neuro-pediatric facilities, and were submitted to a detailed headache questionnaire, which consisted of demographic and clinical data. To evaluate social anxiety, the Social Phobia Inventory was used. A total of 151 subjects were evaluated: 50 had chronic migraine, 50 had episodic migraine and 51 were control subjects. In the chronic migraine group, the mean score in the Social Phobia Inventory was $18.5 \pm 12.4$, which was significantly higher than in the episodic migraine group $(12.1 \pm 8.1)$ and in the control group (13.8 \pm 10.8 ; $\mathrm{F} 2131=4.8, \mathrm{P}=0.010)$. The mean score, however, was not significantly different between the control and episodic migraine groups. Chronic migraine is strongly associated with high social anxiety score, regardless of demographic data and pain intensity. The total burden of migraine may be increased with social anxiety disorder comorbidity.

Del Carmen Ospina-Ospina (2011). The study was conducted on anxiety and depression in adolescents between 10 to 17 year-old .This study was aimed at estimating the prevalence of symptoms of depression and anxiety amongst 10 to 17 year-old adolescents still attending school

(C) The International Journal of Indian Psychology, ISSN 2348-5396 (e)| ISSN: 2349-3429 (p) | 52 


\section{Anxiety among Adolscents and Adults}

using the CDI and SCARED questionnaires for early screening. This was a cross-sectional study of prevalence. $37 \%$ of the 538 adolescents interviewed had anxiety symptoms, $12.3 \%$ had depression symptoms and $9 \%$ presented anxiety and depression symptoms, males having greater prevalence for depression symptoms (6.9 \% cf $5.4 \%$ for females) but lacking statistical significance. There was a greater tendency for anxiety symptoms to be found in adolescents attending public schools. Concerning co morbidity, more anxiety symptoms were found in adolescents having depression symptoms. It was concluded that anxiety and depression symptoms are real at this age, this being reason enough why it is necessary to suspect and detect them on time so that adolescents can receive suitable attention.

LorahD.Dorn(2008)The study was conducted among differences in depressive symptoms and anxiety between (a) normal weight and overweight, and (b) morning type and evening type (sleep chronotype) adolescent girls. The interaction of sleep chrono type and weight and depressive symptoms and anxiety were also examined. The design consisted of a cross-sectional study of 264 adolescent females (mean age $=14.9 \pm 2.2$, range 11-17 years). Sleep chronotype, depressive symptoms, and anxiety were obtained by self-report questionnaire. Compared with normal-weight females, overweight females were more likely to be non-Caucasian, lower socioeconomic status,. sleep and weight impact physical and mental health during adolescence. The combination of evening chronotype and overweight appears to have the strongest association on the emotional health of adolescent females.

Scott Bellini (2008) the study examined the prevalence and types of anxiety exhibited by highfunctioning adolescents with autism spectrum disorders and factors related to this anxiety. Results suggest that adolescents with autism spectrum disorders exhibit anxiety levels that are significantly higher than those of the general population. The study found a low negative correlation between assertive social skills and social anxiety. In addition, a moderate curvilinear relationship was found between empathic skills and the various social anxiety measures. Results of the study support an emerging body of research demonstrating elevated anxiety levels in highfunctioning individuals with autism spectrum disorders.

Robert S. Weinberg and Daniel Gould (2007), "anxiety is a negative emotional state characterized by nervousness, worry, and apprehension and associated with activation or arousal of the body". There are several subcategories of anxiety that one must first be familiar with to better understand what contributes to performance anxiety. Cognitive anxiety is the anxiety that is directly related to our thought processes, because to become anxious we first must have a thought about a negative feeling. Also, somatic anxiety is another aspect that comes into play, which is the physical component. These two components encompass the mental and physical sides associated with anxiety. 


\section{Anxiety among Adolscents and Adults}

Larry L. Mullins, (2000) the study sought to examine dating anxiety and problems in social relationships and health-related quality of life in adolescents and young adults with food allergies compared with their healthy peers. It was hypothesized that individuals with food allergies would experience greater dating anxiety and poorer social functioning and physical and mental healthrelated quality of life than their healthy peers. Participants with food allergies were age, gender, and ethnicity matched to young adults without a history of allergies or any other chronic illness for analyses. The majority of adolescents and young adults with food allergies reported that their allergies interfere with physical intimacy with their current partner. Results further revealed that adolescents and young adults with food allergies reported greater dating anxiety and fear of negative evaluation than healthy peers. No differences were observed between the groups on physical or mental health-related quality of life or social functioning. For both groups, dating anxiety was a significant predictor of mental quality of life and social functioning.

Daniel P Dickstein (1995) the study was conducted on. Anxiety disorders are the most prevalent mental health concern facing adolescents today, yet they are largely undertreated. we focus on the most common types of anxiety disorders, often known as phobias, which include generalized anxiety disorder, social anxiety/social phobia, separation anxiety disorder, panic disorder, and specific phobias. In summary, anxiety is a common psychiatric problem for adolescents, but armed with the right tools, primary care providers can make a major impact.

Rompf and colleagues (1993) report on a study exploring anxiety levels and major concerns among 255 undergraduate and graduate students before starting practicum. They found that undergraduates were more anxious than graduate students; rating themselves an average of 6.4 versus 5.9 on a scale where 1 was "not very anxious" and 10 was "very anxious." Experience with one practicum did not lead to significant differences in anxiety levels among students. The further along students were in their academic program, the better prepared they felt for their placement. However, the more advanced students also tended to be older and have more volunteer or work experience than the undergraduates, and both age and prior experience were positively correlated with feelings of preparedness.

Based on their results, Rompf and colleagues (1993) discuss potential interventions to reduce student anxiety, including instituting formal orientation programs in placements, providing additional information to students about their agency, and training students in time management. They also call for additional research in this area to increase our understanding of students' preplacement anxiety so that social work educators can help manage and decrease it. 


\section{Anxiety among Adolscents and Adults}

Hocevar (1991) examined test anxiety among students in Brazil, Egypt, and the United States. The study found that test anxiety in all three cultures was higher among female students than among male students.

Nils Pearson (1991). Depression and anxiety levels in three groups of children and adolescents, those with learning disabilities (LD), with conduct disorders (CD), and with no disabilities (ND), were measured with the student self-report scale and teacher rating scale of the Depression and Anxiety in Youth Scale. Results revealed highest self-ratings and teacher ratings in both depression and anxiety for the group with CD. Students with LD did not self-rate as more depressed and anxious than children without disabilities but were rated higher in depression by their teachers. Self-rating did not reveal, as teachers' ratings did, a progressive increase in anxiety and depression with age. Females self-rated higher than males in depression and anxiety; however, teachers rated males as more depressed and anxious than females. Special education teachers of students with CD and those with LD were more accurate than general educators in identifying students who self-rated as significantly depressed and/or anxious.

Sharma and Sud (1990) found that female students experience higher levels of test anxiety than do males irrespective of cultural background. The study involved students from four Asian cultures. The conclusion drawn from these findings was that a major causal factor involved in the gender-related differences in test anxiety among students was a greater role expectation conflict among females than among males. Sud (1991) examined test anxiety among students in India and the United States. This study found no differences in levels of test anxiety experienced by female and male students; however, the study did find that, in the United States, levels of test anxiety were higher among high school students of both genders than among college students.

Since Scovel's (1978), several studies have been conducted that consider the role of anxiety in language learning. This paper examines the perspectives from which foreign language anxiety research has been conducted, the instruments that have been used, and the results that have been reported. Three approaches to the study of anxiety are identified as the trait, state, and situation specific perspectives. The instruments chosen to measure anxiety have been quite varied, with several scales specifically intended to assess foreign language anxiety. The literature to be reviewed comes from studies of children, studies that have included anxiety in models of language learning, and finally, studies that are focused directly on the role of anxiety in language learning. With the advances in theory and measurement that have been made in the past decade or so, it is anticipated that foreign language anxiety will receive much more.

Scott Belli. (1937) The present study examined factors contributing to the development of social anxiety in adolescents with autism spectrum disorders (ASD). In particular, the study examined 


\section{Anxiety among Adolscents and Adults}

the contribution of social skill deficits and physiological hyper-arousal to the development of social anxiety in individuals with ASD. Forty-one adolescents with high-functioning ASD participated in the study. The results of a multiple regression analysis suggest that the proposed model (i.e., the combination of social skills and physiological arousal) is a significant predictor of social anxiety in this sample. A developmental pathways model is proposed that identifies potential predisposing or protective factors that may contribute to the development, protection, or remediation of social anxiety in adolescents with autism.

Susan J Bradley, the study was conducted on Anxiety and mood disorders are among the most common disorders in adolescents. The present paper briefly reviews the epidemiology of anxiety and mood disorders in adolescents. Phenomenology is referred to according to the major diagnostic categories for anxiety and depression. Contributing factors, including genetic and environmental components and their possible interaction, are discussed. Anxiety and mood disorders in adolescents are prevalent conditions that may become chronic, and may carry a risk of current and later functional impairment. Their etiology is multi-factorial and often involves a negative interaction of a stress reactive individual within a stress-provoking situation. Treatment goals are stress reduction, exposure to the avoided or feared situation and practicing strategies for anxiety reduction. A broad range of interventions are effective in reducing both anxiety and depression, and need to be tailored to the needs of the child and family.

\section{Aim}

- To measure the anxiety level among male adolescent and adult group.

\section{Hypothesis}

1. Anxiety level is higher in adolescent group as compared to adult group.

\section{Variables}

- Independent variable: - Age

- Dependent variable: - Anxiety level

\section{Design}

Between group design is used for the study.

\section{Sample}

The sample consists of 30 male subjects of which 15 are adolescents and 15 are adults.

\section{Inclusion Criteria}

Only those who are above the age of 18 years and within the age of 25 years are included in the study. 


\section{Anxiety among Adolscents and Adults}

All the subjects in the study are from non-psychology background.

\section{Materials/Tools}

Anxiety scale Questionnaire developed by Kurg, Scheier and R.B.Cattell [IPAT]

Writing material

Scoring key and norms

\section{Procedure}

The subject is seated comfortably. Rapport has to be established. The subject has to be given a copy of the anxiety scale booklet. The subject is asked to answer the questions in the scale. The responses have to be scored with the help of the key and the anxiety level has to be determined. The test is not time limited but typically requires only about 10 minutes for the subject of average reading ability who is not excessively disturbed. Anyone taking an unusually long time should be reminded that 'first responses' to each question is what is needed.

\section{Instructions}

"Inside this booklet there are 40 statements about how people feel or think at one time or another. There is no right or wrong answers. Just pick the one that is really true for you and mark the $\mathrm{a}, \mathrm{b}$ or c answer".

\section{Precautions}

1. Every effort should be made to secure the true answer from the subject.

2. The subject should interpret the questions by himself. The experimenter may give meaning of the questions.

3. All questions should be answered and there has to be only one answer for each question.

\section{Analysis Of Results}

1. The responses are scored using the interpretation sheet and the total and mean is calculated.

2. ' $t$ ' test is been calculated for the series.

\section{SCORING}

Table 1 shows the individual scores of the subjects.

\begin{tabular}{|l|l|l|l|l|}
\hline SI.No & Name & Adults & Name & Adolescents \\
\hline 1 & P.R & 8 & S.B & 9 \\
\hline 2 & A.K.M & 9 & D.J & 8 \\
\hline 3 & S.S & 7 & P.A & 8 \\
\hline 4 & R.K.C & 7 & H.P & 7 \\
\hline 5 & B.K.C & 9 & R.K & 6 \\
\hline 6 & S.K.G & 8 & M.R & 9 \\
\hline 7 & S.K.V & 8 & J.M & 7 \\
\hline
\end{tabular}

(C) The International Journal of Indian Psychology, ISSN 2348-5396 (e)| ISSN: 2349-3429 (p) | 57 
Anxiety among Adolscents and Adults

\begin{tabular}{|l|l|l|l|l|}
\hline SI.No & Name & Adults & Name & Adolescents \\
\hline 8 & A.N.K & 7 & M.G.V & 8 \\
\hline 9 & M.S & 8 & M.S.M & 10 \\
\hline 10 & D.V.M & 9 & M.K & 8 \\
\hline 11 & I.S & 8 & B.A.B & 8 \\
\hline 12 & D.C.Y & 7 & S.M.Y & 10 \\
\hline 13 & A.S & 8 & S.K & 8 \\
\hline 14 & M.I & 8 & Y.U & 9 \\
\hline 15 & K.V & 7 & M.S & 9 \\
\hline TOTAL & & 118 & & 124 \\
\hline MEAN & & 7.86 & & 8.26 \\
\hline
\end{tabular}

Table 2 shows the total, mean, SD and $t$ scores.

\begin{tabular}{|l|l|l|}
\hline Groups & Adults & Adolescents \\
\hline TOTAL & 118 & 124 \\
\hline MEAN & 7.86 & 8.26 \\
\hline EX2 & 6.31 & 16.82 \\
\hline SD1 AND SD2 & 0.67 & 1.09 \\
\hline SEM1 AND SEM2 & 0.17 & 0.29 \\
\hline SED & 0.31 & \\
\hline 't' & 1.29 & \\
\hline
\end{tabular}

Graph 1 : Showing the following mean scores for adults and adolescents.

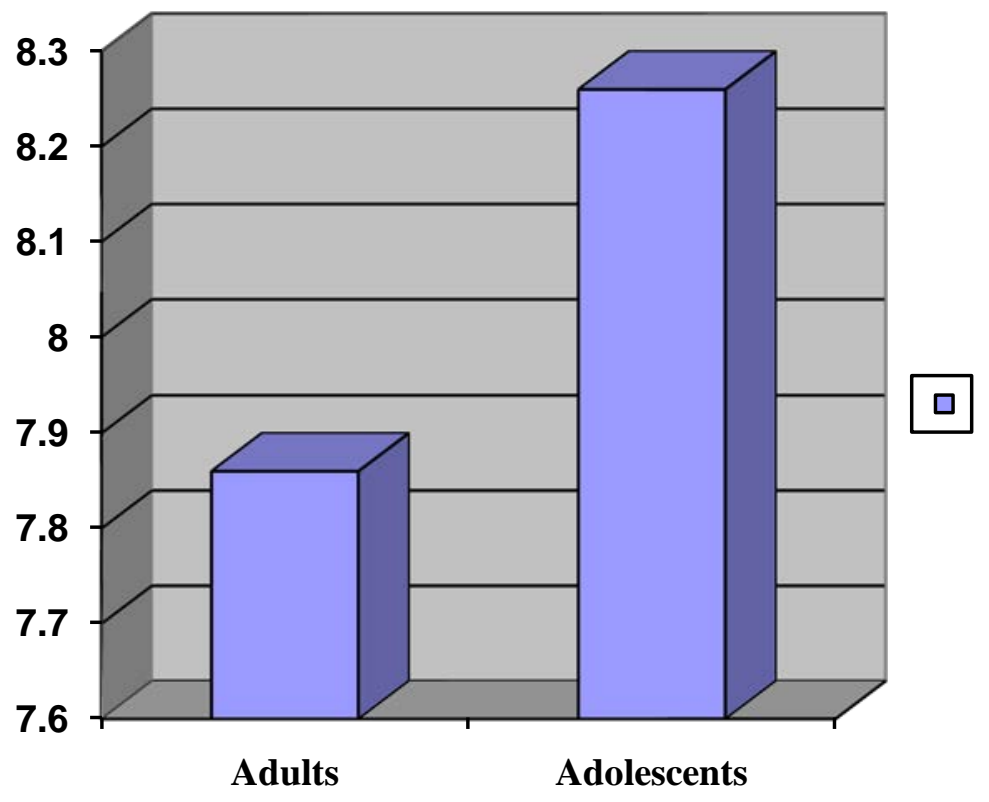

(C) The International Journal of Indian Psychology, ISSN 2348-5396 (e)| ISSN: 2349-3429 (p) | 58 


\section{Anxiety among Adolscents and Adults}

\section{SUMMARY}

The aim of the experiment was to measure the anxiety level among male adolescent and adult group. It was hypothesized that anxiety level is higher in adolescent group as compared to adult group. The experiment was conducted on 30 male subjects, 15 adolescents and 15 adults. All within the age group of 18 to 25 .

Table 2 shows the mean, SD and ' $t$ ' scores of adolescent and adult group. Looking at the table it can be seen that the adolescent group has a mean of 8.26 and SD of 1.09, the adult group has a mean of 7.86 and SD of 0.67 and the obtained ' $t$ ' score is 1.29 hence the adolescent group proves the hypothesis which states that anxiety level is higher in adolescent group as compared to adult group.

The obtained ' $\mathrm{t}$ ' score is 1.29. which is not significant at both 0.05 and 0.01 level. Hence, when we measure the anxiety among adolescent and adult group. Adolescent have more anxiety when compared to adult group, it can be because adults are more adjusted towards the environment as compared to adolescent group.

\section{CONCLUSION}

The purpose of the study was to measure the anxiety level among adolescent group and adult group. It was hypothesized that adolescent group anxiety level is higher in adolescent group as compared to adult group. The sample used in the study was 30 male subjects out of which 15 were adolescents and 15 adults of age group between 18 to 25. The study was conducted on medical students of INDIAN INSTITUTE OF AYURVEDIC MEDICINE AND RESEARCH. Between group design was used for the study. The tools used for the study was Self Analysis Form [IPAT] developed by Kurg, Scheier and R.B.Cattell the questionnaire had 40 questions with 3 alternatives $A, B, C$. The data was analyzed using ' $t$ ' test the obtained ' $t$ ' scores were 1.29 which is not significant at both 0.05 and 0.01 level.

Thus, the adolescent group proves the hypothesis which states that "anxiety level is higher in adolescent group as compared to adult group".

\section{Acknowledgments}

The author appreciates all those who participated in the study and helped to facilitate the research process.

\section{Conflict of Interests}

The author declared no conflict of interests.

\section{REFERENCES}

Bhatnagar, S., \& Saxena, A. (2000). Advanced Educational Psychology. Meerut: Surya Publications.

Boring, Langfield.,\& Weld. (1963). Foundation of Psychology. Bombay: Asia Publishing House.

(c) The International Journal of Indian Psychology, ISSN 2348-5396 (e)| ISSN: 2349-3429 (p) | 59 


\section{Anxiety among Adolscents and Adults}

Bradley, Robert H.; Corwyn. Robert, F. (1995). Home Environment and Behavioural Development during Early adolescence. Psychology and mental health Publication. Merrill Palmer Quarterly.

Bryme, B. (2000). Relationship between Anxiety, Fear, Self Esteem and Coping Strategies. Journal of Educational psychology. Vol 35(137): 201-215.

Campbell, M. (2004). Teaching Tips for the Prevention of Anxiety in Children. Brisbane: QLD:QSS.

Deb, S. (2001, October). A study on the negative effects of academic stress. Paper presented at the International Seminar on Learning and Motivation, Kedah Darul Aman, Malaysia.

Lindzey, Gardner., \& Mcintyre. (1991). Psychology. New York.

Ravi, M., \& Latha. (2005). A study of perceived family environment in Relation to adjustment and Academic Achievement. Psychological Studies, 44, pp 66-75.

Revina, A.M., Gregory, M.G.,\& Franklin, Caroline, J. S.(2014). Test Anxiety Levels of Board Exam Going Students in Tamil Nadu, India: Bio Med Research International Volume 2014 pp. 1-9, .

Robinson, E.H., Rotter, J.C., Fey, M.A., \& Vogel, K.R. (1992). Helping Children Cope with Fears and Stress. Ann Arbor, MI: University of Michigan.

How to cite this article: Pavithraraj, Chandramohan V (2016), Anxiety among Adolscents and Adults, International Journal of Indian Psychology, Volume 4, Issue 1, No. 80, ISSN:2348-5396 (e), ISSN:2349-3429 (p), DIP:18.01.106/20160401, ISBN:978-1-365-57867-0 